\title{
Papers
}

\section{Cost effectiveness and cost utility model of public place defibrillators in improving survival after prehospital cardiopulmonary arrest}

\author{
Andrew Walker, Jane M Sirel, Andrew K Marsden, Stuart M Cobbe, Jill P Pell
}

\begin{abstract}
Objective To determine the cost effectiveness and cost utility of locating defibrillators in all major airports, railway stations, and bus stations throughout Scotland.

Design Economic modelling exercise with data from Heartstart (Scotland). Parameters used in economic model included direct costs derived for increased accident and emergency attendances, increased hospital bed days, purchase and maintenance of defibrillators, and training in their use; life years gained calculated from increased discharges from hospital and mean survival after discharge; utility (quality of life) obtained from published data. Sensitivity analyses tested the robustness of model. Future gains discounted at $1.5 \%$ a year and future costs at $6 \%$.

Setting Whole of Scotland.

Subjects Records of all prehospital cardiac arrests due to presumed heart disease that occurred in a major airport, railway, or bus station between May 1991 and March 1998 and were not witnessed by ambulance or medical staff.

Main outcome measures Observed survival to hospital admission and observed survival to discharge. Predicted survival calculated by applying observed survival in patients attended by ambulance staff within three minutes to those who waited longer.

Results The total discounted direct costs were $£ 18325$ a year. The cost per life year gained was £29 625 (\$49 625, €43 151) and the cost per quality adjusted life year (QALY) gained was $£ 41146$ (\$68 924, €59 932). More widespread provision of public place defibrillators would increase these figures.

Conclusions The cost per QALY calculated for public place defibrillators represents poorer value for money than some alternative strategies for improving survival after prehospital cardiopulmonary arrest, such as the use of other trained first responders. The figure exceeds the commonly discussed cut off levels for funding in the United Kingdom and United States of $£ 30000$ and $\$ 50000$ per QALY, respectively.
\end{abstract}

\section{Introduction}

Defibrillation improves survival after prehospital cardiopulmonary arrest. The proportion of people with arrhythmias that can be treated by defibrillation decreases rapidly with time. One strategy for reducing time to defibrillation is the location of automated external defibrillators in public places that can be used by non-healthcare personnel (for example, untrained bystanders and trained lay members of the public such as airport staff) before the emergency medical services arrive.
Increasing numbers of defibrillators are being provided in public places in the United Kingdom ${ }^{1}$ and the United States. ${ }^{2}$ However, there is little information on the value for money of such a strategy. Without such information it is impossible to determine whether greater benefit could be achieved by investing the same amount of money in alternative strategies, such as reduced response times for the emergency medical service, and provision of defibrillators to other first responders, such as members of the fire and police services.

In England, the Department of Health has committed more than $£ 3 \mathrm{~m}$ to provide defibrillators in 72 public places. In contrast, no central funding has, as yet, been provided for public place defibrillators in Scotland. We determined the economic efficiency of locating defibrillators in all major airports, railway stations, and bus stations throughout Scotland.

\section{Methods}

\section{Data source}

Scotland has a total population of 5.1 million in urban, semirural, and rural areas. The Scottish Ambulance Service is the sole emergency prehospital ambulance service. Since 1988, ambulance crews have collected data prospectively on all resuscitation attempts after cardiopulmonary arrest. The data include call-response interval (time from telephone call to arrival of the ambulance), location of the arrest, and whether defibrillation was attempted. Since 1991, the presumed cause of arrest has been classified according to the Utstein convention. ${ }^{3}$ Staff in hospital medical records departments document whether patients are discharged alive. The data are collated to form the Heartstart (Scotland) register.

\section{Economic model}

We considered the status quo (no public place defibrillators) and the provision of defibrillators in all major airports and railway and bus stations. We compared costs and benefits from a health service perspective. In clinical trials, outcome is usually measured as survival, though quality adjusted survival is also important to patients and decision makers. Therefore, we undertook a cost effectiveness analysis using life years gained and a cost utility analysis using quality adjusted life years (QALY) gained.

During the period studied, there were no public place defibrillators in Scotland. All prehospital defibrillation was undertaken by ambulance staff (99\%) or general practitioners $(1 \%)$. We identified all arrests that occurred in a major airport or railway or bus station over seven years from May 1991 and were due to cardiac disease and were not witnessed by ambulance staff. We determined the observed survival to admission and discharge and predicted survival after location of defibrillators in 
these sites. A proportion of patients are unsuitable for defibrillation because their initial arrthymia is asystole or pulseless electrical activity. Therefore, we assumed that public place defibrillators could, at best, improve survival compared with that obtained by early attendance of ambulance staff. In the sites studied we applied the observed survival among patients attended by ambulance staff within three minutes to those who waited longer to calculate predicted survival. This method is described in greater detail elsewhere. ${ }^{4}$ We calculated current mean survival after discharge from hospital and applied this to the additional patients who could be discharged alive. We applied a measure of utility (quality of life) derived from a study that used the health utilities index mark III. ${ }^{5}$

In the basic model, we included the direct costs to the health service, including purchase and maintenance of automated external defibrillators, basic training of key staff members located nearby, and the marginal costs resulting from additional or prolonged hospital admissions. The ambulance costs of travelling to the arrest site and transferring the patient away were assumed to be equivalent in both groups. We applied the average cost for attendance at an accident and emergency department. For additional patients admitted to hospital, we applied a mean length of stay of three days in a coronary care unit before transfer to a general medical or cardiology ward. We applied average Scottish NHS costs to these beds. ${ }^{6}$ We assumed that there would be no direct costs generated by the additional survivors requiring outpatient attendance or readmission after discharge. Also, we included no indirect costs-for example, due to social care or employment. All costs were based on data for the financial year April 2000 to March 2001, inclusive.

Costs and benefits were estimated for the average period of survival after arrest. We adopted usual economic practice and placed less weight on future gains. We adhered to the National Institute for Clinical Excellence (NICE) recommendations by discounting future costs and benefits at $6 \%$ and $1.5 \%$ a year, respectively. The cost effectiveness and cost utility ratios were calculated by dividing the difference in discounted lifetime costs between the two options by the difference in discounted lifetime health benefits.

\section{Sensitivity analyses}

To test the robustness of the basic model, we varied the assumptions and values used in a series of sensitivity analyses. We considered several one way sensitivity analyses including both evidence based and speculative changes, as listed below. The evidence based changes that produced maximum effect were then combined in a series of multi-way sensitivity analyses.

Health benefits-Because the number of arrests was small, we applied upper and lower limits derived from the $95 \%$ confidence intervals. We assumed that the effectiveness of public place defibrillators was equivalent to the effectiveness of early ambulance attendance. However, both public place defibrillators and early ambulance attendance may produce improvements in management additional to early defibrillation. To test the sensitivity of the model to the assumptions made regarding effectiveness, we examined the effect of the survival gains (to accident and emergency, admission, and discharge) being 25\% higher and 25\% lower than predicted. We examined the impact of the higher utility value $(0.93)$ published by Kuntz et al. ${ }^{7}$

Defibrillator cost-As with other technologies, the purchase cost of defibrillators is likely to decrease with time. We assessed the impact of a $50 \%$ reduction. Our basic model assumed that the number of defibrillators per site was sufficient to enable prompt defibrillation of everyone arresting in that site. The association between number of defibrillators and increase in survival is unlikely to be linear, and there may be a threshold effect. Because of lack of information on this association we were unable to model the effect of providing fewer defibrillators per site than required to provide complete coverage. However, we did model the possibility that we could achieve complete coverage, and therefore equivalent benefit, using fewer defibrillators (total $21 v 31$ ).

Hospital costs-In the basic model we used published hospital costs. The daily inpatient cost of $£ 234$ is derived from both general medical and cardiology beds. The latter are more expensive. Therefore, in the sensitivity analysis, we used the higher cost of $£ 460$ calculated by Netten et al. ${ }^{8}$ The accident and emergency costs are based on all patients presenting, including for minor trauma. Patients attending after cardiopulmonary arrest require above average resources. We modelled the impact of an arbitrary 10 -fold increase. Because of lack of data in the basic model we assumed that additional survivors would not generate long term costs due to clinic attendances, hospital admissions, or drug therapies. In the sensitivity analyses we included an arbitrary additional $£ 5000$ per patient.

Discount rates-As recommended by NICE, we modelled the impact of discounting benefits at $0 \%$ and $6 \%$.

\section{Results}

\section{Numbers of sites, arrests, and defibrillators}

Over the period studied, there were four major airports, nine major railway stations, and four major bus stations in Scotland, equating to 17 eligible sites. Over seven years, 38 arrests occurred in these locations, equating to 5.4 a year. In the basic model, we assumed that four defibrillators would be located in each airport, one in each bus station, two in each of the largest two railway stations, and one in each of the seven others (31 defibrillators altogether).

\section{Admission, survival, and quality of life}

The observed survival to arrival at the accident and emergency department, admission, and discharge was $52.9 \%, 26.4 \%$, and $14.7 \%$, respectively. If public place defibrillators were available, the predicted survival figures were $66.7 \%, 33.3 \%$, and $16.7 \%$, respectively. Therefore, the marginal increases a year in the numbers surviving were $0.7(13.8 \%), 0.4(6.9 \%)$, and $0.1(2.0 \%)$, respectively.

The mean length of stay after admission was 24 days among those discharged alive, 8 days among those who died, and 14 days overall. Application of these figures to the 5.4 eligible arrests a year resulted in 0.7 additional patients attending accident and emergency and 5.6 additional inpatient days; 1.4 in coronary care and 4.2 in general medicine or cardiology. Mean survival after discharge alive from hospital was 6.2 years.

After discounting, we gained 5.7 life years per survivor-that is, surviving to discharge from hospital. We applied the utility value of 0.72 , calculated by Nichol et al, ${ }^{5}$ to estimate the gain in QALYs. This meant that, on average, health related quality of life after arrest was $72 \%$ of normal. The gain in QALYs per survivor was, therefore, 4.5 undiscounted and 4.1 discounted.

\section{Costs}

The mean costs were $£ 46$ per accident and emergency attendance, £612 per day in coronary care, and £234 per day in general medicine or cardiology. ${ }^{6}$ Each defibrillator cost $£ 2500$ to buy and maintain, which was converted into the capital charge that a health service budget holder would pay. Training was calculated per site rather than per defibrillator. We applied the costs 
Table 1 One way sensitivity analyses of effect of public place defibrillators

\begin{tabular}{|c|c|c|c|c|}
\hline & Original & Changed to & Cost / QALY & Change \\
\hline Basic model & & & $£ 41146$ & \\
\hline \multicolumn{5}{|l|}{ Changes made to QALYs: } \\
\hline No of arrests in Scotland over 7 years & 38 & 26 & $£ 58302$ & $42 \%$ \\
\hline No of arrests in Scotland over 7 years & 38 & 50 & $£ 32225$ & $-22 \%$ \\
\hline Survival gains increased by $25 \%$ (A\&E, ward, discharge) & $13.8 \%, 6.9 \%, 2.0 \%$ & $17.3 \%, 8.6 \%, 2.5 \%$ & $£ 26568$ & $-35 \%$ \\
\hline Survival gains reduced by $25 \%$ (A\&E, ward, discharge) & $13.8 \%, 6.9 \%, 2.0 \%$ & $10.4 \%, 5.2 \%, 1.5 \%$ & $£ 53549$ & $30 \%$ \\
\hline Utility of survivors & 0.72 & 0.93 & $£ 31855$ & $-23 \%$ \\
\hline Discount applied to health benefits & $1.5 \%$ & $0 \%$ & $£ 39069$ & $-5 \%$ \\
\hline Discount applied to health benefits & $1.5 \%$ & $6 \%$ & $£ 47671$ & $16 \%$ \\
\hline \multicolumn{5}{|l|}{ Changes made to costs of defibrillators: } \\
\hline No of defibrillators (but same benefit) & 31 & 21 & $£ 29699$ & $-28 \%$ \\
\hline Unit cost of defibrillators & $£ 2500$ & $£ 1250$ & $£ 23403$ & $-43 \%$ \\
\hline \multicolumn{5}{|l|}{ Changes made to treatment costs: } \\
\hline Ward costs & $£ 234$ & $£ 460$ & $£ 42096$ & $2 \%$ \\
\hline Accident and emergency costs & $£ 46$ & $£ 460$ & $£ 41842$ & $2 \%$ \\
\hline Length of hospital stay of survivors & 24 days & 16 days & $£ 40690$ & $-1 \%$ \\
\hline Length of CCU stay of those who die in hospital & 3 days & 8 days & $£ 43720$ & $6 \%$ \\
\hline Lifetime NHS costs after discharge & $£ 0$ & $£ 5000$ & $£ 42365$ & $3 \%$ \\
\hline
\end{tabular}

$\mathrm{QALY}=$ quality adjusted life year, $\mathrm{A \& E}=$ accident and emergency department, $C C U$ coronary care unit.

Table 2 Multi-way sensitivity analyses of effect of public place defibrillators

\begin{tabular}{|c|c|c|c|c|}
\hline & Original & Changed to & Cost/QALY & Change \\
\hline Basic model & & & $£ 41146$ & \\
\hline \multicolumn{5}{|l|}{ Increase in QALYs: } \\
\hline No of arrests in Scotland over 7 years & 38 & 50 & $£ 23689$ & $-42 \%$ \\
\hline Utility of survivors & 0.72 & 0.93 & & \\
\hline Discount applied to health benefits & $1.5 \%$ & $0 \%$ & & \\
\hline \multicolumn{5}{|l|}{ Decrease in QALYS: } \\
\hline No of arrests in Scotland over 7 years & 38 & 26 & $£ 67548$ & $64 \%$ \\
\hline Discount applied to health benefits & $1.5 \%$ & $6 \%$ & & \\
\hline \multicolumn{5}{|l|}{ Reduction in costs of defibrillators: } \\
\hline No of defibrillators (but same benefit) & 31 & 21 & $£ 17679$ & $-57 \%$ \\
\hline Unit cost of defibrillators & $£ 2500$ & $£ 1250$ & & \\
\hline \multicolumn{5}{|c|}{ Increase in QALYs plus reduction in defibrillator costs: } \\
\hline No of arrests in Scotland over 7 years & 38 & 50 & $£ 13776$ & $-67 \%$ \\
\hline Utility of survivors & 0.72 & 0.93 & & \\
\hline Discount applied to health benefits & $1.5 \%$ & $0 \%$ & & \\
\hline Unit cost of defibrillators & $£ 2500$ & $£ 1250$ & & \\
\hline
\end{tabular}

of the Scottish Ambulance Service training course, which was $£ 52.40$ a year (A Marsden, Scottish Ambulance Service, personal communication). The life expectancy of a defibrillator was five years (A Marsden, Scottish Ambulance Service, personal communication).

The overall initial capital cost of the defibrillators was $£ 77500$. The capital charge over five years was $£ 93310$ without discounting and $£ 79020$ with discounting at 6\%. Including training, this equated to $£ 16554$ a year. The incremental cost due to additional accident and emergency attendances and in hospital care was $£ 1770$ a year. Therefore, the total direct cost combining capital and revenue was $£ 18325$ a year. The life year gain per year was 0.72 , hence the discounted net cost per life year gained was $£ 29625$. The QALY gain a year was 0.44 , producing a discounted net cost per QALY gained of $£ 41146$.

\section{Sensitivity analyses}

Table 1 contains the results of the one way analyses. The cost effectiveness ratio produced by the basic model had a small denominator and large numerator. Therefore, the results were more sensitive to assumptions relating to health gain than cost. The results were particularly resistant to changes in hospital costs. Even extreme changes, such as doubling ward costs and multiplying accident and emergency costs by 10 , had minimal impact.

In the multi-way analyses, combination of changes that impacted favourably on health gain produced a net cost per QALY of $£ 23686$ (table 2). Combination of assumptions with the opposite effect produced a figure of $£ 67548$. Application of both assumptions resulting in reduced defibrillator cost produced a figure of $£ 17679$. The most extreme scenario, including the most favourable assumptions about both cost and outcome, produced a figure of $£ 13776$.

\section{Discussion}

Public place defibrillators may not be the most cost effective means of improving survival and quality of life after prehospital arrest. Alternative strategies, such as use of trained first responders, may produce greater improvements and better value for money (table 3). However, caution is needed when we compare studies from countries with different healthcare systems. Also, the sensitivity analyses showed that our results were sensitive to assumptions regarding effectiveness. Therefore, our conclusions require corroboration from prospective studies. In England, the Department of Health has provided funding for 700 public place 
Table 3 Cost effectiveness of other population based interventions for prehospital cardiopulmonary arrest

\begin{tabular}{|c|c|c|}
\hline Intervention & Cost $^{*} /$ QALY & Economics methods: discount rate $(\%) \dagger$ \\
\hline Reduction in response time of single tier EMS ${ }^{9}$ & $£ 384252$ & 5 \\
\hline Reduction in response time of two tier EMS (midpoint) $)^{9}$ & $£ 110681$ & 5 \\
\hline Change from one tier to two tier EMS (midpoint) ${ }^{9}$ & $£ 69959$ & 5 \\
\hline Other trained first responder (police) ${ }^{5}$ & $£ 24534$ & 3 \\
\hline Community CPR programmes $^{10}$ & $£ 213939$ & Discount rate and time horizon not clear \\
\hline \multicolumn{3}{|l|}{ Automated external defibrillators on: } \\
\hline Large commercial aircraft (>200 passengers) ${ }^{11}$ & $£ 26195$ & 3 \\
\hline Medium and large commercial aircraft ( $\geq 100$ passengers $)^{11}$ & $£ 56471$ & 3 \\
\hline All commercial aircraft ${ }^{11}$ & $£ 126745$ & 3 \\
\hline
\end{tabular}

QALY=quality adjusted life year, EMS=emergency medical service, $C P R=$ cardiopulmonary resuscitation.

${ }^{*} £ 1=\$ 1.56$; includes allowance for inflation at $5 \%$ a year.

tCosts after discharge not considered in any of these studies.

defibrillators. ${ }^{1}$ The American Heart Association supports the principle ${ }^{12}$ but advised that expansion should be halted until further information on clinical and cost effectiveness from clinical trials is available. ${ }^{2}$

The Heartstart register collects data prospectively throughout Scotland and therefore provides an invaluable tool for modelling the potential impact of public place defibrillators. In a previous study we showed that only $21 \%$ of arrests not witnessed by ambulance crews occurred in sites where public place defibrillators could impact on survival and only then if defibrillators were as commonplace as fire extinguishers. ${ }^{4}$ Furthermore, this subgroup already had the best profile in terms of call-response interval, defibrillation, and survival and therefore the least scope for improvement. We calculated that locating defibrillators in every public site across Scotland would increase overall survival from $5.0 \%$ to $6.5 \%{ }^{4}$ This is less than the potential improvement achievable through some alternative strategies. ${ }^{13}$

\section{Cost analysis}

In the present study we also carried out complementary cost analyses. We are aware of only two previous cost analyses on public place defibrillators. Both were limited by a lack of data on either cost or outcome. ${ }^{514}$ The Heartstart register provided us with accurate information on both the location and outcome of arrest, enabling us to make informed calculations of the potential cost, coverage, and impact of public place defibrillators.

In our model we included sites similar to those selected by the Department of Health, such as major airports and railway stations. England covers about 130410 square kilometres (50 351 square miles). The Department of Health has provided funding for 700 defibrillators in 72 sites across the whole of England. Woollard attempted to model the cost effectiveness of this strategy. ${ }^{14}$ A crucial assumption in his model was that half of all future arrests in public places in the whole of England will occur in the 72 sites in which defibrillators have been located. Because airports have a high volume of human traffic they are considered good locations for defibrillators. However, they often cover a large area, covering several levels, and the people using them are relatively young and mobile. Gratton et al reviewed all arrests over one year in Kansas City. ${ }^{15}$ Kansas City is urban and fairly densely populated. It has 430000 residents and covers 834 square kilometres (322 square miles). The 326 arrests occurred in 288 different locations. Of the 16 locations where more than one arrest occurred, only five were public places and only two arrests occurred in each site. Kansas City airport covers 10 hectares (25 square acres) and the two arrests at the airport occurred $6.1 \mathrm{~km}$ (4 miles) apart. Gratton et al concluded that "nursing homes" are the only non-healthcare sites in which the incidence and density of arrests justifies provision of defibrillators. ${ }^{15}$
In the cost effectiveness study conducted by Nichol et al, they assumed that the "density of distribution of defibrillators ... was such that one device was available for each cardiopulmonary arrest that occurred in public." However, they acknowledged having no data on the costs needed to achieve this level of coverage. They included an incremental cost per arrest of only $\$ 1200$ for public place defibrillators compared with $\$ 3300$ for police first responders. Only $\$ 300$ of the police cost was attributed to training. The location of future arrests cannot be predicted accurately. Public place defibrillators are commonly carried on foot and therefore can cover only a small area. By contrast, police vehicles can transport defibrillators rapidly to more than one site. Hence, public place defibrillators have to be distributed more densely to achieve the same level of coverage as a dynamically deployed system. Therefore, it is difficult to conceive how the cost per arrest of providing "one device ... for each cardiac arrest that occurred in public" could be achieved more cheaply with public place defibrillators than with police first responders.

\section{Relevance of extrapolation}

In our study, the survival gain from public place defibrillators was estimated from a comprehensive population based register because randomised controlled trial data were not available. We were obliged to use a figure for utility obtained from a different population which may or may not be applicable to Scotland. ${ }^{5}$ We attempted to compare our results with other population based strategies for reducing time to defibrillation. However, caution should be used in the comparison of results from studies undertaken in different countries or at different points in time. Conversions can take account of currency exchange rates and inflation but do not take account of differences in current service provision, population distribution, or frequency of arrest.

We obtained a cost per QALY gained of $£ 41146$ (\$68 924) for providing defibrillators in all major airports and railway and bus stations. Expansion beyond these sites is likely to reduce cost effectiveness. Although there is no absolute "cut off" for funding, the chairman of the National Institute for Clinical Excellence (NICE) observed that recommended technologies tend to cost $£ 30000$ per QALY or less. This figure corresponds well to the cut off of $\$ 50000$ frequently applied in the United States. The figure we obtained from our basic model exceeds these values. The sensitivity analyses identified factors that could potentially bring this figure below the funding threshold, such as a reduction in the purchase cost of defibrillators.

It is important to consider the opportunity cost of investing public funding in public place defibrillators. Our study and others indicate that alternative population strategies for reducing time to defibrillation, such as use of trained first responders, might be more clinically ${ }^{40}$ and cost effective. ${ }^{5}$ Implantable 


\section{What is already known on this topic}

Early defibrillation improves survival after prehospital arrest

Public place defibrillators can reduce time to defibrillation in some arrests

Information is lacking on whether greater benefit could be gained by investing the money spent on public place defibrillators in alternative strategies

\section{What this paper adds}

Modelling of costs of locating defibrillators in all major airports, railway stations, and bus stations throughout Scotland resulted in costs of $£ 29625$ for each life year gained and $£ 41146$ for each QALY

These costs represent poorer value for money than some alternative strategies, such as the use of other trained first responders, and exceed the commonly used cut-off levels for funding

cardiac defibrillators are an effective intervention in patients known to be at high risk of cardiopulmonary arrest with a cost per life year gained of $£ 26000-£ 31000 .{ }^{16}$ From a wider perspective, several primary and secondary prevention interventions may be more cost effective in reducing overall mortality from coronary heart disease. ${ }^{17}{ }^{18}$ We support the view of the American Heart Association that evidence of clinical and cost effectiveness from randomised controlled trials is required before further expansion of public place defibrillators can be justified.

We thank the Scottish Ambulance Service and hospital medical records staff for supplying the data on which the study was based.

Contributors: JPP had the original idea for the study and drafted and revised the manuscript.JPP and JMS calculated the observed and predicted survival figures. AW undertook the economics analyses. JPP and AW interpreted the results. AKM provided information on costs of buying defibrillators and training. All authors read and approved the final version. SMC manages the Heartstart register. JPP is the guarantor.

Funding: British Heart Foundation.

Competing interests: None declared.

Ethical approval: Not required.

1 Secretary of State for Health. Saving lives: our healthier nation. London: Department of Health, 1999
2 Nichol G, Hallstrom AP, Kerber R, Moss AJ, Ornato JP, Palmer D, et al. American Heart Association report on the second public access defibrillation conference, April 17-19, 1997. Circulation 1998;97:1309-14.

3 Cummins RO, Chamberlain DA, Abramson NS, Allen M, Baskett PJ, Becker L, et al. Recommended guidelines for uniform reporting of data from out-of-hospital cardiac arrest: the Utstein style. A statement for health professionals from a task force of the American Heart Association, the European Resuscitation Council, the Heart and Stroke Foundation of Canada, and the Australian Resuscitation Council. Circulation 1991;84:960-75

4 Pell JP, Sirel JM, Marsden AK, Ford I, Walker N, Cobbe SM. Potential impact of public access defibrillators on overall survival after out of hospital cardiopulmonary arrest: retrospective cohort study. $B M J$ 2002;325:515-7.

5 Nichol G, Hallstrom A, Ornato J, Riegal B, Stiell IG, Valenzuela T, et al. Potential costeffectiveness of public access defibrillation in the United States. Circulation effectiveness of public access defibrillation in the United States. Circulation 1998;97:1315-20

6 Information and Statistics Division. Scottish health service costs. Edinburgh: NHS Scotland, 2001.

7 Kuntz K, Tsevat J, Goldman L. Cost-effectiveness of routine coronary angiography after acute myocardial infarction. Circulation 1996;94:957-65.

8 Netten A, Rees A, Harrison G. Unit costs of health and social care 2001.University of Kent, Canterbury: Personal Social Services Research Unit, 2002. www.kent.ac.uk/PSSRU/

9 Nichol G, Laupacis A, Stiell I, O'Rourke K, Anis A, Bolley H, et al. Cost-effectiveness analysis of potential improvements to emergency medical services for victims of analysis of potential improvements to emergency medical
out-of-hospital cardiac arrest. Ann Emerg Med 1996;27:711-20.

10 Lee K, Angus D, Abramson N. Cardiopulmonary resuscitation: what cost to cheat death? Crit Care Med 1996;24:2046-52.

11 Groeneveld P, Kwong J, Liu Y, Rodriguez A, Jones M, Sanders G, et al. Cost-effectiveness of automated external defibrillators on airlines. JAMA 2001;28:1482-9.

12 Weisfeldt ML, Kerber RE, McGoldrick RP, Moss AJ, Nichol G, Ornata JP, et al. Public access defibrillation: a statement for health care professionals from the American Heart Association task force on automatic external defibrillation. Circulation 1995;92:2763.

13 Pell JP, Sirel JM, Marsden AK, Ford I, Cobbe SM. Effect of reducing ambulance response times on deaths from out of hospital cardiac arrest: cohort study. BMJ 2001;322:1385-8.

14 Woollard M. Public access defibrillators: a shocking idea? J Public Health Med

2001;23:98-102.
15 Gratton M, Lindholm DJ, Campbell JP. Public access defibrillators. Prehosp Emerg Care 1999;3:303-5.

16 National Institute for Clinical Excellence. Guidance on implantable cardioverter defibrillators for arrthymias. September 2000. www.nice.org.uk/pdf/ Nice+DEFIBRILATOR++guidance.pdf (accessed 15 Mar 2003).

17 Hummel S, Piercy J, Wright R, Davie A, Bagust A, McMurray J. An economic analysis of the survival and ventricular enlargement (SAVE) study. Application to the United Kingdom. Pharmacoeconomics 1997; 12:182-92.

18 Pickin DM, McCabe CJ, Ramsay LE, Payne N, Haq ICS, Yeo WW, et al. Cost effectiveness of HMG-CoA reductase inhibitor treatment related to the risk of coronary heart disease and cost of drug treatment. Heart 1999;82:325-32.

(Accepted 30 September 2003)

bmj.com 2003;327:1316

Robertson Centre for Biostatistics, University of Glasgow, Glasgow G12 8QQ Andrew Walker senior lecturer in health economics

Department of Medical Cardiology, University of Glasgow, Glasgow G32 2ER Jane M Sirel research assistant

Stuart M Cobbe Walton professor of medical cardiology

Scottish Ambulance Service Headquarters, Edinburgh EH10 5UU

Andrew K Marsden medical director

Greater Glasgow NHS Board, Glasgow G3 8YZ

Jill P Pell consultant in public health medicine

Correspondence to: J Pell

jill.pell@gghb.scot.nhs.uk 